\title{
Help-seeking behaviour for sexual problems: the Global Study of Sexual Attitudes and Behaviors
}

\author{
E.D. MOREIRA Jr., ${ }^{1}$ G. BROCK, ${ }^{2}$ D.B. GlaSSER, ${ }^{3}$ A. NICOLOSI,${ }^{4,5}$ E.O. LAUMANN,${ }^{6}$ A. PAIK,${ }^{7}$ \\ T. WANG ${ }^{6}{ }^{\text {C. GINGELL }}{ }^{8}$ FOR THE GSSAB INVESTIGATORS' GROUP. \\ Gonçalo Moniz Research Center, ${ }^{I}$ Oswaldo Cruz Foundation and Diretoria Cientifica, Hospital São Rafael, Salvador, Brazil, St \\ Joseph's Health Care London, ${ }^{2}$ Ontario, Canada, Pfizer Inc, ${ }^{3}$ New York, NY, USA, Department of Epidemiology, ${ }^{4}$ Institute of \\ Biomedical Technologies, National Research Council, Milan, Italy, G.H. Sergievsky Center, ${ }^{5}$ School of Public Health, Columbia \\ University, New York, NY, USA, University of Chicago, ${ }^{6}$ Chicago, IL, USA; University of Iowa, ${ }^{7}$ Iowa City, IA, USA; Southmead \\ Hospital, ${ }^{8}$ Bristol, UK
}

\section{SUMMARY}

The Global Study of Sexual Attitudes and Behaviors (GSSAB) investigated various aspects of sex and relationships among 27,500 men and women aged 40-80 years. Here, we report help-seeking behaviours for sexual problems in this population. A questionnaire was administered using the accepted survey method in each country. Although almost half of all sexually active respondents had experienced at least one sexual problem, less than $19 \%$ of them $(18.0 \%$ of men and $18.8 \%$ of women) had attempted to seek medical help

\section{INTRODUCTION}

Many epidemiological studies of sexual behaviour and disorders have been conducted during the past 10 years in various countries. Reports from these studies have mainly highlighted the prevalence and the severity of sexual problems, such as erectile dysfunction (ED) and disorders of ejaculation or orgasm (1-9). However, there are few studies on the helpseeking behaviour for sexual problems among men and women (10-12); so little is currently known about how gender, psychosocial states and cultural factors might influence help-seeking patterns for sexual problems.

The identification of factors associated with help-seeking patterns among men and women with sexual problems is of interest for clinicians. It may help them to recognise and overcome potential barriers that their patients might have in discussing and seeking help for sexual problems. In addition, the negative impact of such problems on quality of life is now widely recognised $(2,13)$, and thus a better understanding of

\section{Correspondence to:}

Edson D. Moreira Jr, MD, PhD, Unit of Epidemiology and Biostatistics, Oswald Cruz Foundation, Rua Waldemar Falcão 121, Salvador, Bahia, Brazil 40.295-001

Tel.: $+55713568781 \times 243$

Fax: +55713562155

Email: edson@cpqgm.fiocruz.br for their problem(s). The most frequent action taken by men and women was to talk to their partner (39\%). Only $9 \%$ of men and women had been asked about their sexual health by a doctor in a routine visit during the past 3 years. Although sexual problems are highly prevalent, few men and women seek medical help for these problems. Overall, men and women show similar help-seeking behaviours.

Keywords: Survey; treatment; men; women; sexual health; sexual activity; sexual problems

(C) 2005 Blackwell Publishing Ltd sexual health issues regarding help-seeking behaviour may provide a means to improve access to treatment and reduce human suffering.

In this analysis, data from the Global Study of Sexual Attitudes and Behaviors (GSSAB) have been used to describe help-seeking behaviour with regard to six specific sexual problems among men and women aged 40-80 years in 29 countries, representing most of the regions of the world.

\section{METHODS}

The GSSAB is the first large, multicountry survey to systematically study attitudes, beliefs and health in sexual relationships in middle-aged and older adults. The survey involved 27,500 women and men, aged 40-80 years, in 29 countries. We attempted to include countries representing many world regions; no particular criterion was employed to select them, but the convenience for the logistics of the survey.

\section{Data Collection and Sampling}

The study methods employed in the GSSAB have been described in detail elsewhere $(8,9)$. In brief, sampling was based on random-digit-dialing, and data were collected by telephone interview in European (Austria, Belgium, France, Germany, Italy, Spain, Sweden and the UK), North American (Canada and the USA) countries, Australia, New 
Zealand, Israel and Brazil. In Middle Eastern countries and South Africa, sampling was based on a door-to-door protocol, while in East and South-east Asian countries, an intercept protocol was employed, with the exception of Japan, where a questionnaire was sent by mail to a random sample. In all these countries, the data were gathered through selfcompleted questionnaires, with the exception of Mexico, where a mixed-mode method of self-completed questionnaires and telephone interviews was employed. Both the door-to-door and intercept protocols represent accepted survey methods for each country, but are likely to be more reflective of their urban populations. The mean response rates for the telephone interviews and the self-completed questionnaires were 16 and $34 \%$, respectively.

The presence of sexual problems was assessed by asking the following question: 'During the last 12 months have you ever experienced any of the following for a period of 2 months or more when you (i) lacked interest in having sex; (ii) were unable to reach climax (experience orgasm); (iii) reached climax (experienced orgasm) too quickly; (iv) experienced physical pain during sex; (v) did not find sex pleasurable; (vi) had trouble achieving or maintaining an erection (men only); and (vii) had trouble becoming adequately lubricated (women only)?'. Respondents were permitted to answer yes to all that applied. For those indicating the presence of a specific sexual problem, the relative severity was assessed in a followup question: 'For each of these experiences, how often would you say this has occurred during the last 12 months? Would you say that this has occurred occasionally, sometimes, or frequently?'. We considered sexual dysfunctions only the sexual problems that were persistent and at least moderately frequent (i.e. we excluded the individuals who reported occasional problems).

Help-seeking behaviour of the six specific sexual problems was ascertained using two questions. The first question asked respondents to indicate ways in which they had sought any help for the sexual problems they had experienced. The listed options included: 'Talked to partner', 'Talked to a medical doctor (other than a psychiatrist)', 'Looked for information in books or magazines', 'Talked to family member or friend', 'Taken prescription drugs/devices', 'Taken nonprescription drugs', 'Looked for information via the internet', 'Talked to psychiatrist or psychologist', 'Talked to a marriage counsellor', 'Talked to a clergy person or religious adviser', 'Called a telephone help line', 'Other - please specify'. Those who did not report that they had 'Talked to a medical doctor' were then asked 'Why didn't you talk to a doctor about any sexual problems you may have experienced?' and offered a list of 14 possible reasons (from which they were to check all that applied). The reasons included attitudes and beliefs regarding the sexual problem and the patient-doctor relationship.

All respondents (irrespective of whether they reported any sexual problems) were also asked 'During a routine office visit or consultation in the past 3 years, has your physician asked you about possible sexual difficulties without you bringing it up first?' (Yes/No) and 'Do you think a doctor should routinely ask patients about their sexual function?' (Yes/No).

Information regarding education and income were recorded following the national systems of schooling and currencies. We recorded educational level into three categories: 'primary school or less' including subjects with no schooling and those who completed primary school only, 'secondary/high school' including subjects with some secondary/high school education or those who completed high school, and 'at least some college' including people who attended college or university whether or not they achieved a degree. The 'low', 'medium' and 'high' categorisation of household income is relative to each country's standards, in order to allow the comparison of nations with very different absolute mean incomes.

\section{Statistical Analysis}

In the present analysis, the countries were grouped by taking account of geographical region, cultural background and mode of data collection, along the following scheme: Northern Europe: Austria, Belgium, Germany, Sweden and the UK; Southern Europe: France, Israel, Italy and Spain; nonEuropean West: Australia, Canada, New Zealand, South Africa and the USA; Central/South America: Brazil and Mexico; Middle East: Algeria, Egypt, Morocco and Turkey; East Asia: China (including Hong Kong), Japan, Korea and Taiwan; South-east Asia: Indonesia, Malaysia, Philippines, Singapore and Thailand. Before creating the clusters, we ensured that the countries included in each cluster offered a sufficiently homogeneous picture in terms of demographic characteristics, sexual behaviours and mode of data collection.

The prevalence of sexual problems was calculated by dividing the total number of self-reports for each problem by the total number of gender-relevant respondents that reported having had intercourse at least once in the year prior to being interviewed. The denominator for the calculation of the prevalence of specific help-seeking behaviour was the sum of people who reported at least one sexual problem. Missing values were always excluded from the denominator. The prevalence estimates for each regional cluster were age-standardised using the age distribution of each gender in the entire GSSAB study population.

\section{RESULTS}

\section{Demographics, Social Characteristics and Sexual Activity}

Selected characteristics (demographics, social characteristics and sexual activity) of the 27,500 respondents are summarised by country cluster in Table 1 . Overall, more than $80 \%$ of men and $66 \%$ of women reported that they had had sexual 


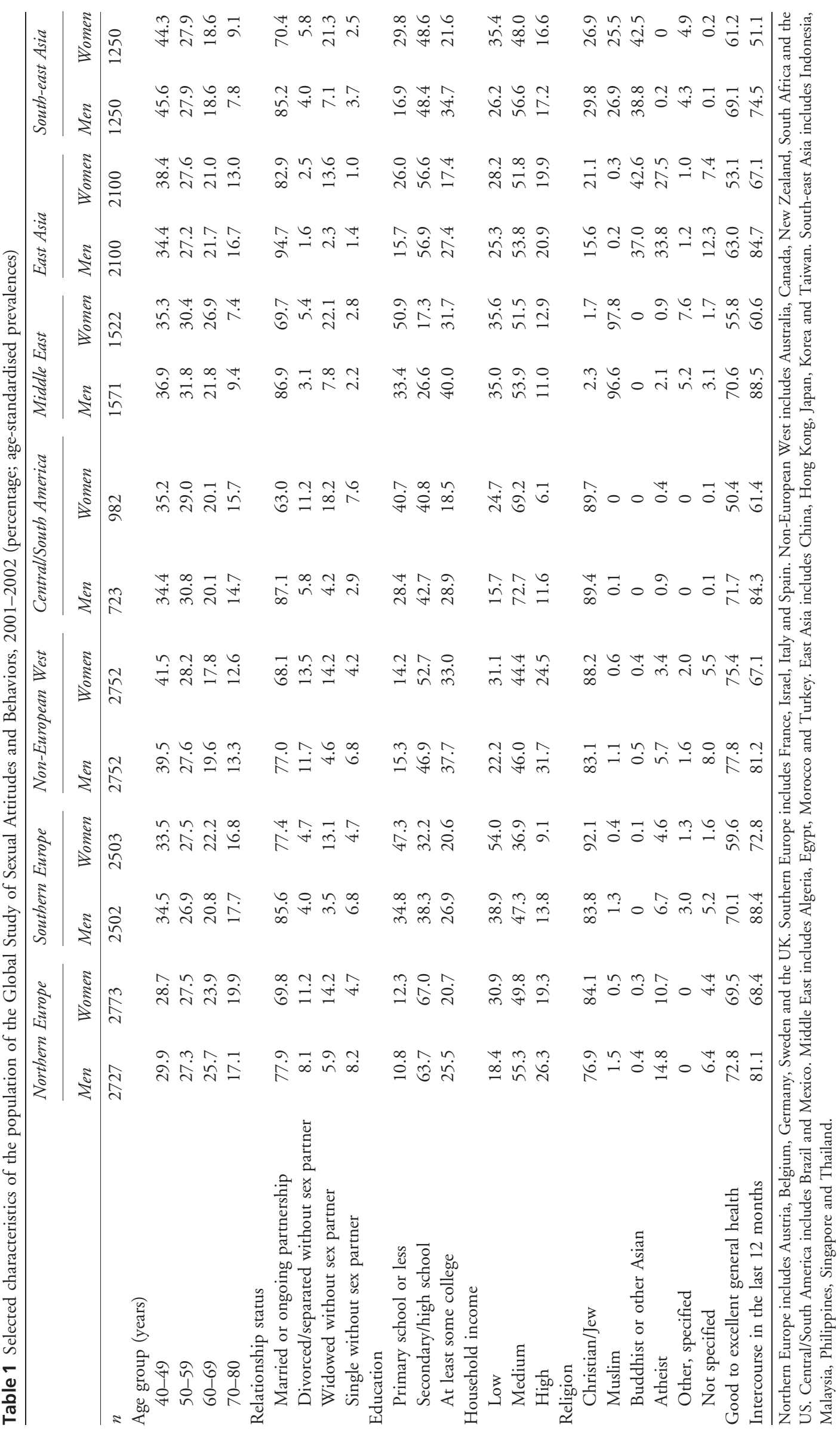


intercourse during the 12 months preceding the interview. Among men, this proportion was $93 \%$ at the age $40-49$ years and $53 \%$ at the age $70-80$ years; among women, it was 88 and $21 \%$, respectively.

Among sexually active respondents, $43 \%$ of men and $49 \%$ of women reported experiencing at least one sexual problem (Table 2). Early ejaculation (24\%) was the most common problem among men, and difficulty in achieving or maintaining an erection (17\%) the second one. Among women, lack of sexual interest was the most common sexual problem (32\%) and inability to reach orgasm the second one (25\%). Experiencing physical pain during intercourse was the least common sexual problem, reported by only $5 \%$ of men and $17 \%$ of women.

\section{Help-Seeking Behaviour for Sexual Problems}

The frequency distribution of help-seeking behaviours for sexual problems among men and women who reported experiencing at least one sexual problem is presented in Table 3. A large percentage of men and women had sought no professional help or advice for their sexual problems, 77.8 and $78.0 \%$, respectively. Only $18.0 \%$ of men and $18.8 \%$ of women had made an attempt to seek medical help. Similarly, $14.6 \%$ of men and $12.8 \%$ of women had used drugs/devices for their problem(s). Men from East Asia were the least likely to seek medical help (6.5\%), whereas those from South-east Asia were the most likely (22.5\%). Among women, those from East Asia were also the least likely to seek medical help (8.5\%), and women from Central/South America were the most likely to do so $(40.8 \%)$.

The most frequent action taken by both men and women (39\% in each case) was 'talk to partner'. Countries from the East Asian and Middle Eastern clusters reported the lowest frequencies and South-east Asia and Southern Europe the highest frequencies of this action (Table 3). Few respondents, $17 \%$ of men and $16 \%$ of women, looked for information in books/magazines or via a telephone helpline or the internet. Of note, men and women from South-east Asia were twice as likely to seek this source of help, 39 and 29\%, respectively (Table 3). On average, 16\% of women and 13\% of men talked to a friend or family member about their sexual problems. Men and women from the South-east Asian cluster reported the highest frequency (29 and 20\%, respectively), while men from Northern Europe (7\%) and women from East Asia (10\%) the lowest frequency of talking to a friend or family member (Table3). The frequency of seeking psychological help (psychiatrist, psychologist or marriage counsellor) was low and varied widely among men $(2-12 \%)$ and women (1-8\%). 'Talk to a clergy person or religious adviser' was the least common action taken by both men (3\%) and women (2\%). However, higher frequencies were reported in countries from the Middle Eastern (by men), Central/South American (by women) and South-east Asian (by both genders) clusters.
Men in Southern European and Non-European West country clusters were more likely to seek medical help for their sexual problems as they got older, a similar trend was observed in Central/South America (Table 4). Among women, in contrast, the likelihood of seeking medical help decreased with age in Southern Europe and South-east Asia and showed a similar tendency in Central/South American and Middle Eastern countries. However, the frequency of this help-seeking behaviour peaked at age 50 and then decreased at older ages in women from the Northern European country cluster, a similar but non-significant increase was observed in Southern European and Non-European West regions (Table 4). The frequency of looking for information anonymously (in books, magazines, telephone helplines or via the internet), talking to a psychiatrist, psychologist or marriage counsellor and talking to a family member or friend generally decreased with age in both genders (data not shown).

Educational attainment was not associated with seeking medical help for sexual problems among men, but women with some college education were more likely to consult with a medical doctor in Northern Europe and Central/South America (Table 4). In general, a higher household income was not correlated with seeking professional help in both genders, except for men from South-east Asian countries and women from Southern European and Central/South American clusters (Table 4). Respondents with higher educational attainment or higher income were more likely to look for information anonymously and to talk to a partner, while subjects with lower income were more likely to talk to a clergy person or religious adviser (data not shown).

Help from a health professional was more likely to be sought by men with erectile difficulties and women reporting lubrication problems (Table 4). Interestingly, inability to reach orgasm in women and lack of sexual interest in both genders did not significantly affect the likelihood of seeking a medical doctor. Men with early ejaculation in Non-European Western and Central/South American countries were less likely to seek medical help for their sexual problems (Table 4).

Respondents who have been asked by a doctor about possible sexual difficulties in a routine visit in the past 3 years or those who think a doctor should routinely ask patients about their sexual function were generally more likely to seek medical help. The same was true for men and women who reported being very-to-somewhat dissatisfied with their sexual function (Table 4). The belief that decreased ability to perform sexually would significantly affect self-esteem was associated with increased probability of seeking medical help among men in some world regions, but not among women. Participants saying that sex was an extremely to very important part of overall life were also more likely to seek medical help (Table 4).

The reasons for not referring themselves to a doctor were similar for men and women (Table 5). The most commonly 


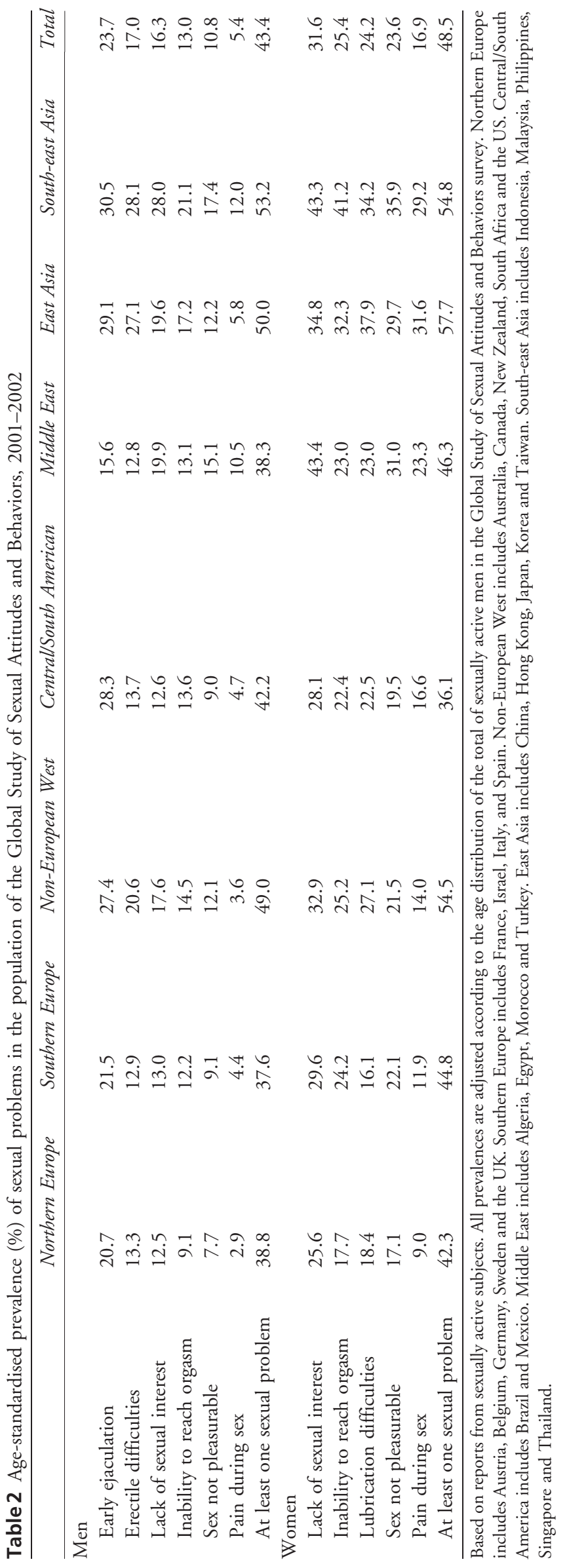




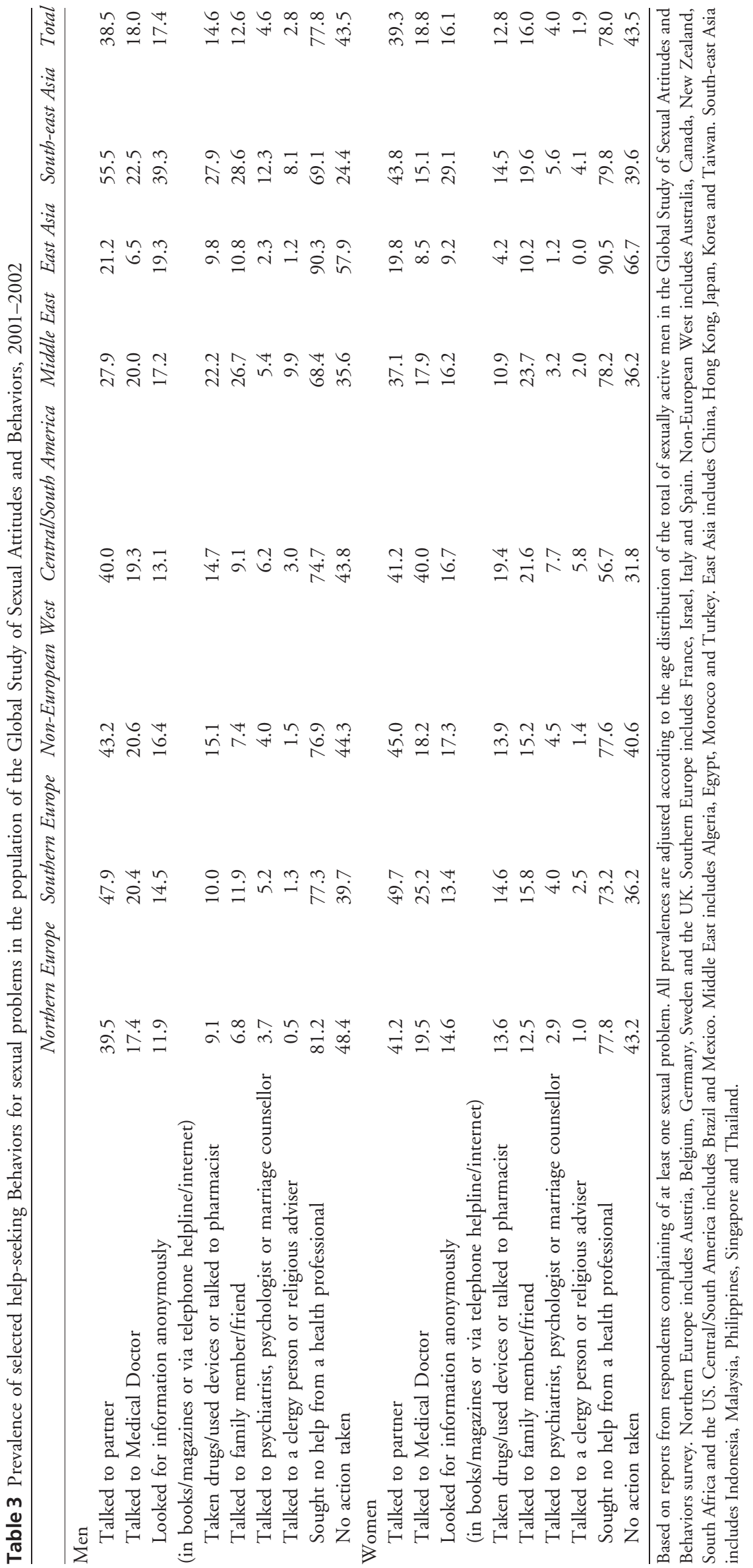




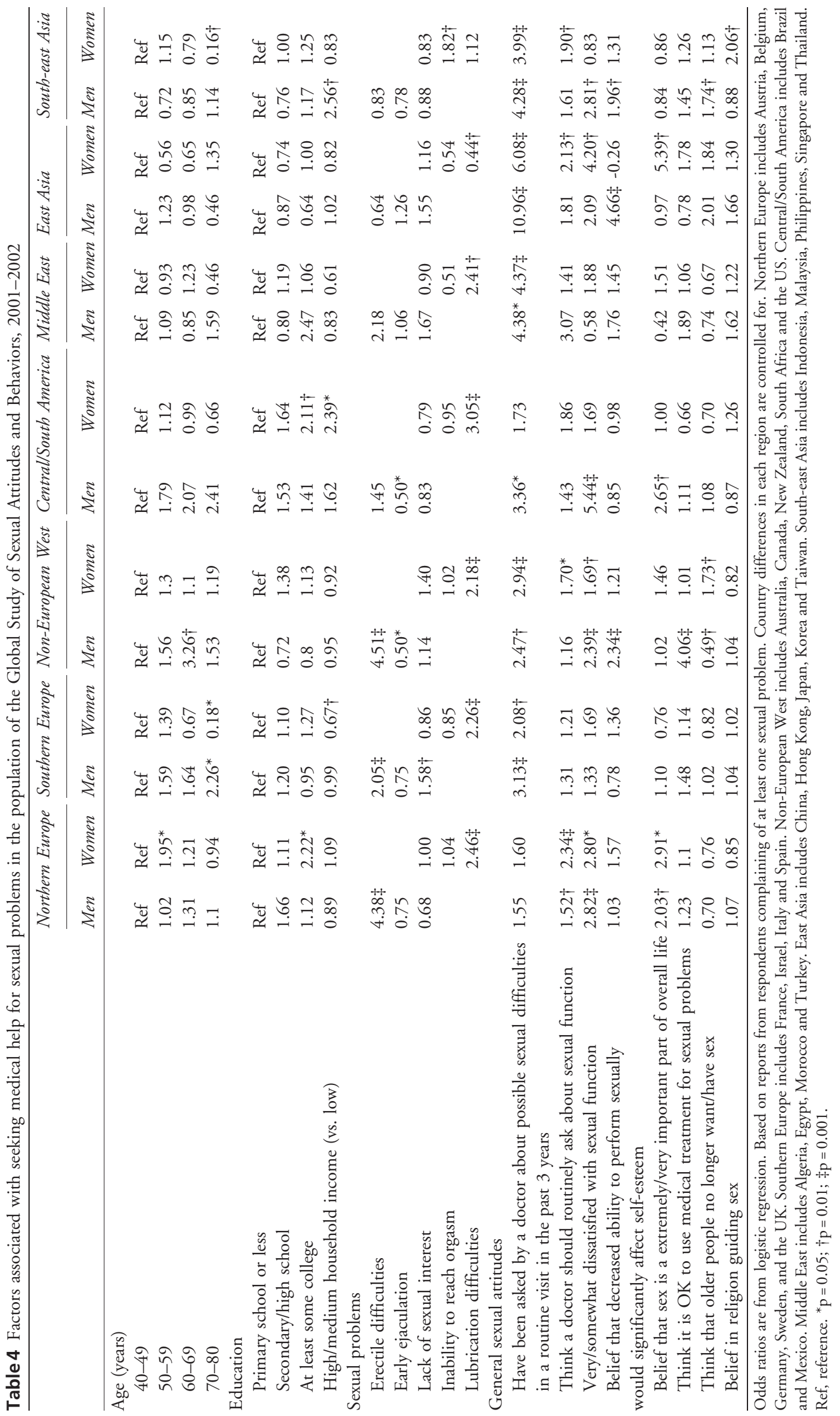




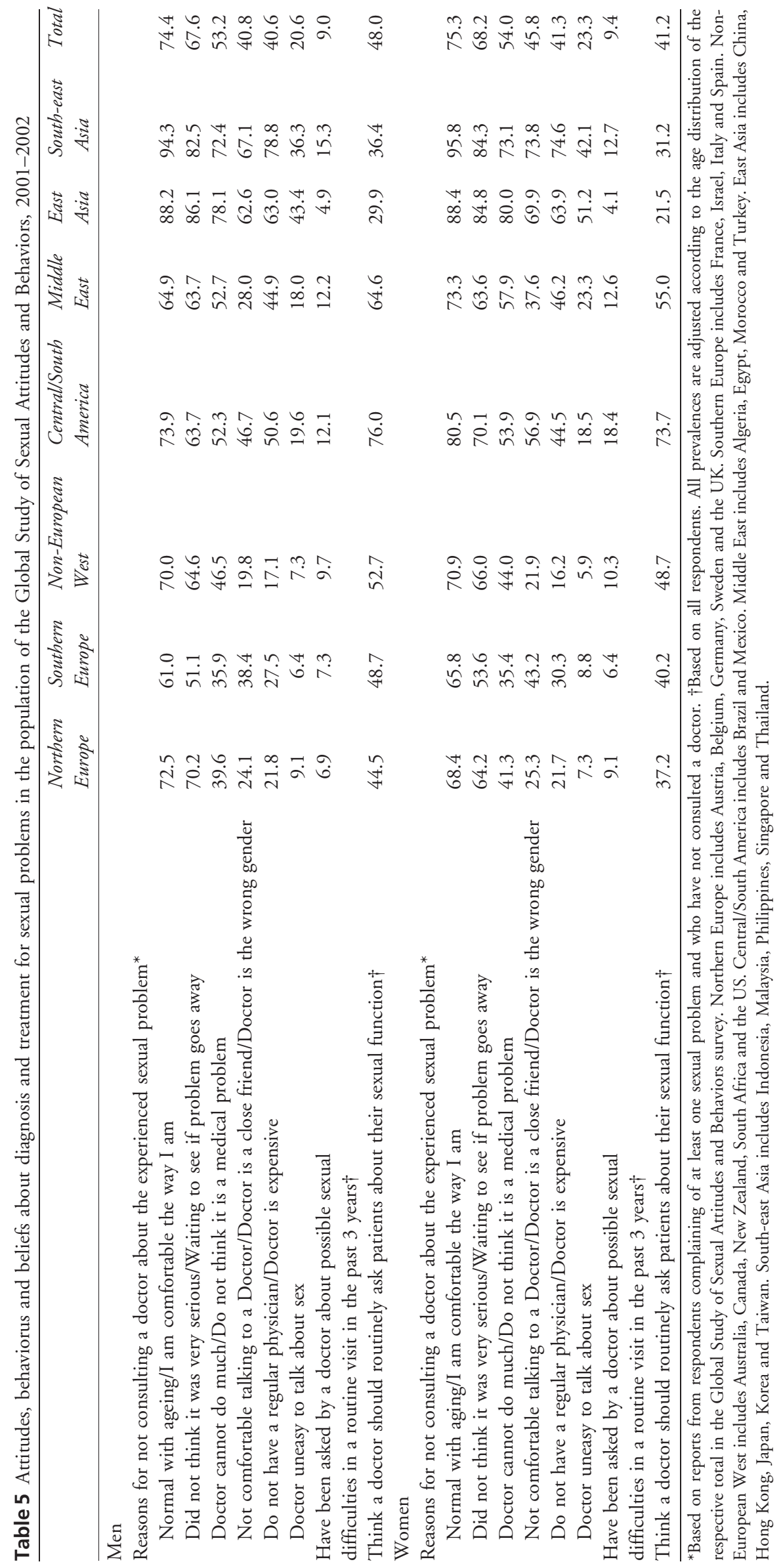


cited reasons were 'I thought it was a normal part of getting older/I am comfortable the way I am' (74\% of men and 75\% of women), and 'I didn't think it was very serious/I was waiting to see if problem goes away' (68\% of both men and women). However, there were important differences between the country clusters and these reasons were cited by a greater proportion of men and women from the East and South-east Asian and, to a lesser extent, Central/South American countries (Table 5). Another set of frequently cited reasons related to the respondent's view of their condition and its treatment: 'I did not think it was a medical problem' and 'I didn't think a doctor could do much for me'. Overall, these reasons were cited by $53 \%$ of men and $54 \%$ of women, but again, there were marked differences between the country clusters, with much higher frequencies in men and women in East and South-east Asian country clusters (72-80\%) compared with the other clusters (35-58\%). The cost of a medical consultation was also cited as an impediment, and its importance was higher to people residing in the South-east Asian, Middle Eastern and Central/South American country clusters (Table 5).

The subjects were also asked whether a doctor had asked them in a routine visit during the last 3 years, about their sexual health. Only $9 \%$ of both men and women answered positively, although $48 \%$ of men and $41 \%$ of women declared that they thought that a doctor should spontaneously ask about sexual problems in the course of a routine consultation. Again, there were variations among the country clusters. Among men, $76 \%$ of the Central/South American cluster thought that a doctor should spontaneously ask about sexual problems, compared with just 29.5\% of the East Asian cluster; among women, the respective percentages were 73 and 21\% (Table 5).

\section{DISCUSSION}

In this research, we examined help-seeking behaviours with regard to specific sexual problems among men and women aged 40-80 years in 29 countries, representing most of the world regions. We used data from the GSSAB, the first comprehensive survey of behaviours, attitudes and beliefs regarding sex, intimacy and relationships among middleaged and older men and women in multiple nations.

While reporting at least one sexual problem was relatively common among men and women, and we found that few of them had sought professional help or advice for their sexual problems. As the more physiological sexual problems erectile and lubrication difficulties - clearly have a significant biological component, it is not surprising that subjects affected by these problems were more likely to seek medical help and that this likelihood increased with their age. In contrast, with other health problems in general, where medical help is usually sought by those in better socio-economic circumstances (14-16), the frequency of seeking medical help for sexual problems in our survey did not vary significantly with respondents' educational attainment and income. This suggests that cultural factors and deeply imbedded health beliefs may play a more decisive role in defining the healthseeking behaviour for sexual problems of men and women than socio-economic factors. In fact, the most commonly cited reasons for not referring themselves to a doctor were similar for both genders and reflect their belief that sexual problems are not medical problems. On the contrary, they are thought by many to be an inevitable part of getting older and therefore, there is not much a doctor could do about them. Furthermore, this conviction is consistent with the medical practices the respondents have experienced, as only $9 \%$ of men and women had been asked by a doctor about possible sexual problems during a routine visit in the past 3 years.

The patterns of help-seeking behaviour for sexual problems were remarkably similar in both genders. The rank order of the most common actions taken by men and women were nearly identical; however, the prevalences of some helpseeking behaviours in both men and women varied considerably across country clusters. This may reflect cultural differences about beliefs and attitudes on sexuality among these regions. So, for instance, countries from the Middle Eastern and South-east Asian clusters, where Islam is the predominant religion, reported the highest frequencies of consulting a religious adviser for sexual problems, while this action was rather rare in the other regions.

The primary advantage of this study is its cross-cultural emphasis. With a sample drawn from 29 countries, we identified several patterns of help-seeking behaviours for sexual problems in multiple regions of the world. Despite these strengths, this study has several limitations. First, while these data are broadly inclusive, they may not be truly representative of each country's entire adult and older populations because of the relatively modest response rates attained in the countries with the random-digit-dialing protocol and because the door-to-door and intercept protocols drew heavily on urban populations. In each country, the sampling method was chosen in order to assure the widest coverage of the population. The response rate was greater in those countries where the request for participation involved a personal contact rather than a telephone call. This was expected, as people are more reluctant to refuse participation when a personal contact is involved than when the request is made by an anonymous caller (17). Second, all of our measures are selfreported, and many are based on responses to single items. Hence, there is likely to be considerable measurement error.

Wherever possible, we have compared the country-specific results of the GSSAB surveys with the findings of published studies conducted in these countries. Although there are a number of methodological differences between the various studies, such as the age range of the men included in the 
analyses and the definition of dysfunction that was applied, the age-adjusted estimates of prevalence of ED in the GSSAB (13 to $28 \%$ ) were similar to those reported in studies conducted in Australia (18), Brazil (3), Egypt (19), France (20), Germany (21), Netherlands (22), Spain (23) and the UK (24). Considerably, higher estimates have been reported in studies from Australia (25), Belgium (26), Brazil $(7,27)$, Finland (28,29), Italy (7), Japan (7), Mexico (30), Morocco (31), Thailand (32), Turkey (33), USA (34-36), and lower estimates in Denmark (13) and Sweden (37). Thus, our prevalence estimates are often lower and thus more conservative than in many of the other studies.

There have been relatively few published papers on helpseeking behaviour in patients with sexual problems; however, these studies have raised interesting issues. The results of a recent web-based survey indicated that although $40 \%$ of women reported that they did not seek help from a physician for sexual problems, 54\% reported that they would like to do so. Respondents reported considerable anxiety and frustration about treatment (12). Among 12,761 men with ED who called a free call information service in Italy between 1997 and 1999, 56.9\% reported that they had discussed their condition with their partner. Overall, half of the men (50.3\%) reported that they had discussed ED with a physician, and the percentage was much higher in men who had ED lasting for more than 3 years $(57.9 \%)$ than in those with ED lasting for less than 6 months (33.6\%). These findings suggest that men may wait some time before discussing their ED with a physician (11). A study of 503 community-based respondents, published in 1990, found that the majority used some form of social help-seeking, favouring informal rather than professional help (10). Furthermore, help-seeking from intimates was associated with obtaining help from other informal contacts. It is interesting to note that more than a decade later, the results of the GSSAB survey indicate that men and women still tend to seek informal rather than professional help for their sexual problems.

\section{CONCLUSION}

Although a relatively high percentage of respondents reported at least one sexual problem and approximately half of both men and women were sufficiently bothered by their problems to talk about them to a partner, friend or family member, only a few sought professional help. It is noteworthy that the pattern of help-seeking behaviour was remarkably similar in men and women. The findings of this international survey should increase awareness and understanding of sexual health issues among clinicians, and help them to identify and overcome potential barriers that their patients' might have in discussing and seeking help for sexual problems. Also, of note is the fact that subjects often think it is the doctor who should begin the discussion of potential sexual problems.

\section{ACKNOWLEDGMENT}

The Global Study on Sexual Attitudes and Behaviors was funded by Pfizer Inc. The authors acknowledge the contribution of their colleagues on the international advisory board for this study: Jacques Buvat (France), Uwe Hartmann (Germany), Sae-Chul Kim (Korea), Rosie King (Australia), Bernard Levinson (South Africa), Ken Marumo (Japan) and Ferruh Simsek (Turkey). The GSSAB was funded by Pfizer Inc.

\section{REFERENCES}

1 Helgason AR, Adolfsson J, Dickman P et al. Sexual desire, erection, orgasm and ejaculatory functions and their importance to elderly Swedish men: a population-based study. Age Ageing 1996; 25: 285-91.

2 Laumann EO, Paik A, Rosen RD. Sexual dysfunction in the United States: prevalence and predictors. JAMA 1999; 281: 537-44.

3 Moreira ED Jr, Abdo CHN, Torres EB et al. Prevalence and correlates of erectile dysfunction: results of the Brazilian Study of Sexual Behavior. Urology 2001; 58: 583-8.

4 Carson CC, West SL, Glasser DB et al. Prevalence and correlates of erectile dysfunction in a United States nationwide populationbased sample: phase I results. J Urol 2002; 167 (Suppl.): 29-30 (Abstract 119).

5 Anastasiadis AG, Davis AR, Ghafar MA et al. The epidemiology and definition of female sexual disorders. World J Urol 2002; 20: 74-8.

6 Carson CC, Glasser DB, Laumann EO et al. Prevalence and correlates of premature ejaculation among men aged 40 years and older: a United States nationwide population-based study. J Urol 2003; 169: 321 (Abstract 1249).

7 Nicolosi A, Moreira ED Jr, Shirai M et al. Epidemiology of erectile dysfunction in four countries: cross-national study of the prevalence and correlates of erectile dysfunction. Urology 2003; 61: 201-6.

8 Laumann EO, Nicolosi A, Glasser D et al. Sexual problems among women and men aged 40-80 years: prevalence and correlates identified in the Global Study of Sexual Attitudes and Behaviors. Int J Impot Res (in press).

9 Nicolosi A, Laumann EO, Glasser D et al. Sexual behavior and sexual problems after the age of 40: the Global Study of Sexual Attitudes and Behaviors. Urology (in press).

10 Catania JA, Pollack L, McDermott LJ et al. Help-seeking behaviors of people with sexual problems. Arch Sex Behav 1990; 19: 235-50.

11 Mirone V, Gentile V, Zizzo G et al. Did men with erectile dysfunction discuss their condition with partner and physicians? A survey of men attending a free call information service. Int $J$ Impot Res 2002; 14: 256-8.

12 Berman L, Berman J, Felder $S$ et al. Seeking help for sexual function complaints: what gynecologists need to know about the female patient's experience. Fertil Steril 2003; 79: 572-6.

13 Ventegodt S. Sex and the quality of life in Denmark. Arch Sexual Behav 1998; 27: 295-307. 
14 Elofsson S, Unden AL, Krakau I. Patient charges - a hindrance to financially and psychosocially disadvantage groups seeking care. Soc Sci Med 1998; 46: 1375-80.

15 Lannin DR, Mathews HF, Mitchell J et al. Impacting cultural attitudes in African-American women to decrease breast cancer mortality. Am J Surg 2002; 184: 418-23.

16 Bussing R, Zima BT, Gary FA et al. Barriers to detection and help-seeking, and service use for children with ADHD symptoms. J Behav Health Serv Res 2003; 30: 176-89.

17 Weinhardt LS, Forsyth AD, Carey MP et al. Reliability and validity of self-report measures of HIV-related sexual behavior: progress since 1990 and recommendations for research and practice. Arch Sex Behav 1998; 27: 155-80.

18 Richters J, Grulich AE, de Visser RO et al. Sex in Australia: sexual difficulties in a representative sample of adults. Aust NZJ Public Health 2003; 27: 164-70.

19 Seyam RM, Albakry A, Ghobish A et al. Prevalence of erectile dysfunction and its correlates in Egypt: a community-based study. Int J Impot Res 2003; 15: 237-45.

20 Guiliano F, Chevret-Measson M, Tsatsaris A et al. Prevalence of erectile dysfunction in France: results of an epidemiological survey of a representative sample of 1004 men. Eur Urol 2002; 42: 382-9.

21 Braun M, Wassmer G, Klotz T et al. Epidemiology of erectile dysfunction: results of the 'Cologne Male Survey'. Int J Impot Res 2000; 12: 305-11.

22 Blanker MH, Bosch JLHR, Groeneveld FPMJ et al. Erectile and ejaculatory dysfunction in a community-based sample of men 50-78 years old: prevalence, concern, and relation to sexual activity. Urology 2001; 57: 763-8.

23 Martin-Morales A, Sanches-Cruz JJ, de Tejada IE et al. Prevalence and independent risk factors for erectile dysfunction in Spain: results of the Epidemiologia de la Disfuncion Erectil Masculina Study. J Urol 2001; 166: 569-75.

24 Dunn KM, Croft PR, Hackett GI. Sexual problems: a study of the prevalence and need for health care in the general population. Fam Pract 1998; 15: 519-24.

25 Pinnock CB, Stapleton AM, Marshall VR. Erectile dysfunction in the community: a prevalence study. Med J Aust 1999; 171: 353-7.
26 Mak R, de Backer G, Kornitzer M et al. Prevalence and correlates of erectile dysfunction in a population-based study in Belgium. Eur Urol 2002; 41: 132-8.

27 Moreira ED Jr, Lisboa Lôbo CF, Villa M et al. Prevalence and correlates of erectile dysfunction in Salvador, northeastern Brazil: a population-based study. Int J Impot Res 2002; 14 (Suppl. 2): S3-S9.

28 Koskimäki J, Hakama M, Huhtala $\mathrm{H}$ et al. Effect of erectile dysfunction on frequency of intercourse: a population based prevalence study in Finland. J Urol 2000; 164: 367-70.

29 Shiri R, Koskimäki J, Hakama M et al. Prevalence and severity of erectile dysfunction in 50-75-year-old Finnish men. J Urol 2003; 170: 2342-4.

30 Ugarte y Romano F, Aguirre JB. Prevalencia de disfunción eréctil en México y factores de riesgo asociados. Rev Mex Urol 2001; 61: 63-76.

31 Berrada S, Kadri N, Mechakra-Tahiri S et al. Prevalence of erectile dysfunction and its correlates: a population-based study in Morocco. Int J Impot Res 2003; 15 (Suppl. 1): S3-7.

32 Kongkanand A and the Thai Erectile Dysfunction Epidemiology Study Group. Prevalence of erectile dysfunction in Thailand. Int $J$ Androl 2000; 23 (Suppl. 2): 77-80.

33 Akkus E, Kadiolglu A, Esen A et al. (Turkish Erectile Dysfunction Prevalence Study Group). Prevalence and correlates of erectile dysfunction in Turkey: a population-based study. Eur Urol 2002; 41: 298-304.

34 Bacon CG, Mittleman MA, Kawachi I et al. Sexual function in men older than 50 years of age: results from the Health Professionals Follow-up Study. Ann Intern Med 2003; 139: 161-8.

35 Feldman HA, Goldstein I, Hatzichristou DG et al. Impotence and its medical and psychosocial correlates: results of the Massachusetts Male Aging Study. J Urol 1994; 151: 54-61.

36 Ansong KS, Lewis C, Jenkins P et al. Epidemiology of erectile dysfunction: a community-based study in rural New York state. Ann Epidemiol 2000; 10: 293-6.

37 Fugl-Meyer AR, Fugl-Meyer KS. Sexual disabilities, problems and satisfaction in 18-74 year old Swedes. Scand J Sexol 1999; 2: 79-105.

Paper received May 2004, accepted August 2004 